\title{
Inflammatory myoglandular polyp as a cause of recurrent abdominal pain in a patient with quiescent Crohn's disease
}

\author{
Aikaterini Mantakaa , Dimitrios P. Bouklas ${ }^{b}$, Erasmia Vlachouc ${ }^{c}$ loannis Grammatikakis ${ }^{d}$, \\ Ioannis Papaconstantinou ${ }^{e}$, Dina G. Tiniakos ${ }^{b, f}$, Evaggelia Anagnostopoulou ${ }^{a}$
}

"Saint George" General Hospital of Chania, Crete, Greece; Aretaieion Hospital, Medical School, National and Kapodistrian University of Athens, Greece; Army Share Fund Hospital (NIMTS), Athens, Greece; University Hospital of Heraklion, Crete, Greece; Newcastle University, Newcastle upon Tyne, UK

\section{Abstract}

Chronic inflammation of the intestinal mucosa has been associated with the appearance of inflammatory polyps or pseudopolyps. Among the distinct categories of inflammatory polyps are inflammatory myoglandular polyps (IMGP) usually found in the colorectum. Only one case of IMGP in the terminal ileus has been described since their first description. We report the first case of an inflammatory polyp with both hyperplastic and myoglandular histological characteristics, in the terminal ileum of a patient with quiescent Crohn's disease, causing recurrent intussusception.

Keywords Inflammatory myoglandular polyp, inflammatory bowel disease, ileal polyp, Nakamura polyp

Ann Gastroenterol 2021; 34 (x): 1-3

\section{Introduction}

Inflammatory bowel disease (IBD), comprising both Crohn's disease (CD) and ulcerative colitis (UC), is a chronic autoinflammatory disease of the gastrointestinal (GI) tract characterized by recurrent periods of flare and inflammation. This chronic inflammatory process, especially in more severe cases or those not well-controlled by available treatment options, leads to the formation of polypoid mucosal protrusions known as pseudopolyps, or post-inflammatory polyps [1]. These serve

aDepartment of Gastroenterology, "Saint George" General Hospital of Chania, Crete, Greece (Aikaterini Mantaka, Evaggelia Anagnostopoulou); ${ }^{\mathrm{b}}$ Department of Pathology, Aretaieion Hospital, Medical School, National and Kapodistrian University of Athens, Athens, Greece (Dimitrios P. Bouklas, Dina G. Tiniakos); 'Gastroenterology Department, Army Share Fund Hospital (NIMTS), Athens Greece (Erasmia Vlachou); ${ }^{\mathrm{D} D e p a r t m e n t}$ of Radiology,

University Hospital of Heraklion, University of Crete Medical School (Ioannis Grammatikakis, Ioannis Papaconstantinou); ${ }^{2} 2^{\text {nd }}$ Department of Surgery, Areteion Hospital, National and Kapodistrian University of Athens, Athens, Greece (Ioannis Papaconstantinou); ${ }^{\text {TT}}$ Translational and Clinical Research Institute, Faculty of Medical Sciences, Newcastle University, Newcastle upon Tyne, UK (Dina G. Tiniakos)

Conflict of Interest: None

Correspondence to: Aikaterini Mantaka MD, PhD, Department of Gastroenterology, "Saint George" General Hospital of Chania, 73300, Crete, Greece, e-mail: katmant@gmail.com

Received 2 May 2021; accepted 13 July 2021; published online 14 September 2021

DOI: https://doi.org/10.20524/aog.2021.0664 as an indirect marker of the alternating cycles of inflammation and healing of the ulcerated mucosa [1]. Their prevalence varies from $4-74 \%$, with a predominance in UC patients $[2,3]$.

Intussusception, a complication of inflammatory polyps in both UC and CD, has been reported in up to $3 \%$ of patients, mainly in cases of large size [4]. We report the case of a CD patient in remission with recurrent abdominal pain due to a giant inflammatory polyp of the terminal ileum with a rare histological appearance, including both hyperplastic and myoglandular changes, causing intussusception.

\section{Case report}

A 38-year-old male with a 6-year history of ileocolonic and upper GI CD, treated with adalimumab for the previous 2.5 years, complained of acute abdominal pain, mainly in the epigastrium and right upper quadrant, while he was on deep clinical, laboratory and endoscopic remission. During a 3-month period, he was admitted three times to the Emergency Department. Mild abdominal tenderness was present at clinical examination. His laboratory tests and inflammatory markers were always within normal range. An abdominal X-ray showed air-fluid levels of the small bowel. An abdominal computerized tomography performed a few days later, after symptom resolution, excluded inflammation of the terminal ileum, strictures or penetrating complication. A gastroscopy and ileocolonoscopy showed no mucosal lesions in the stomach, duodenum, terminal ileum or colon.

As the episodes became more frequent, a magnetic resonance enterography (MRE) scan was performed to exclude any small 
bowel complication related to CD. MRE revealed a 3.7-cm polyp in the terminal ileum at a distance of $20 \mathrm{~cm}$ from the ileocecal valve (ICV) causing intussusception with partial small bowel obstruction (Fig. 1). A smaller 0.5 -cm polypoid lesion was seen $10 \mathrm{~cm}$ from the ICV. A retrograde double-balloon enteroscopy confirmed the presence of the 2 polyps (Fig. 2). Following this, the patient underwent surgical treatment.

The surgical specimen consisted of a 12.5 -cm-long segment of the terminal ileum, including the 2 aforementioned polyps. Macroscopic examination revealed a sessile polyp with lobulated surface measuring $3.7 \times 3.4 \times 2.6 \mathrm{~cm}$, partially obstructing the ileal lumen. A flat tan mucosal lesion measuring $0.5 \mathrm{~cm}$ in greatest diameter was seen $3 \mathrm{~cm}$ proximally to the polyp.
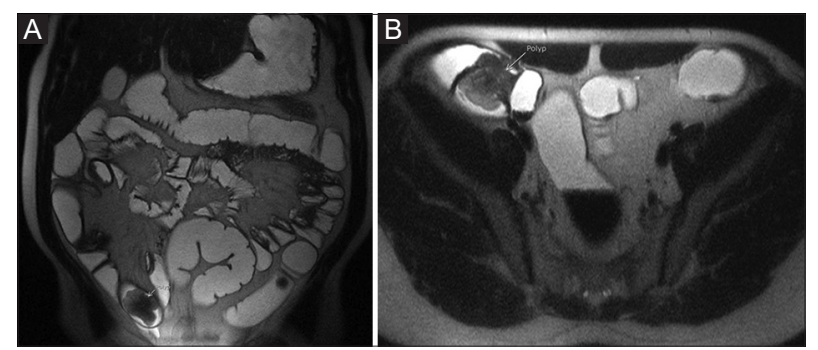

Figure 1 Magnetic resonance enterography. (A) Coronal and (B) axial T2-weighted images. There is a large intraluminal lobulated polypoid mass in the terminal ileum
Histologically, the larger polyp had features of an inflammatory myoglandular polyp, with focal superficial ulceration, rare cryptitis, foci of pyloric metaplasia, and a hyperplastic mesenchymal stroma with vascular proliferation and rare acellular mucin lakes. Immunohistochemistry for $\alpha$ smooth muscle actin and CD68 confirmed the presence of fibromuscular stromal hyperplasia, with numerous proliferating vessels lined by CD31-positive endothelial cells (Fig. 3) and few CD68-positive macrophages. The smaller lesion corresponded to an area of intramural hemorrhage involving the submucosa muscularis propria. No epithelial dysplasia was observed and there was no evidence of cytomegalovirus infection by histology or immunohistochemistry. The surrounding mucosa showed changes consistent with CD in remission.

Nine months post-surgery, the patient is still in clinical, laboratory and endoscopic remission and has gained over $10 \mathrm{~kg}$ in weight.

\section{Discussion}

Inflammatory polyps are the most common type of benign polyps in IBD, although ischemic or infectious colitis, anastomosis and stomas could also be their cause. Subtypes of inflammatory polyps include inflammatory cap polyp,
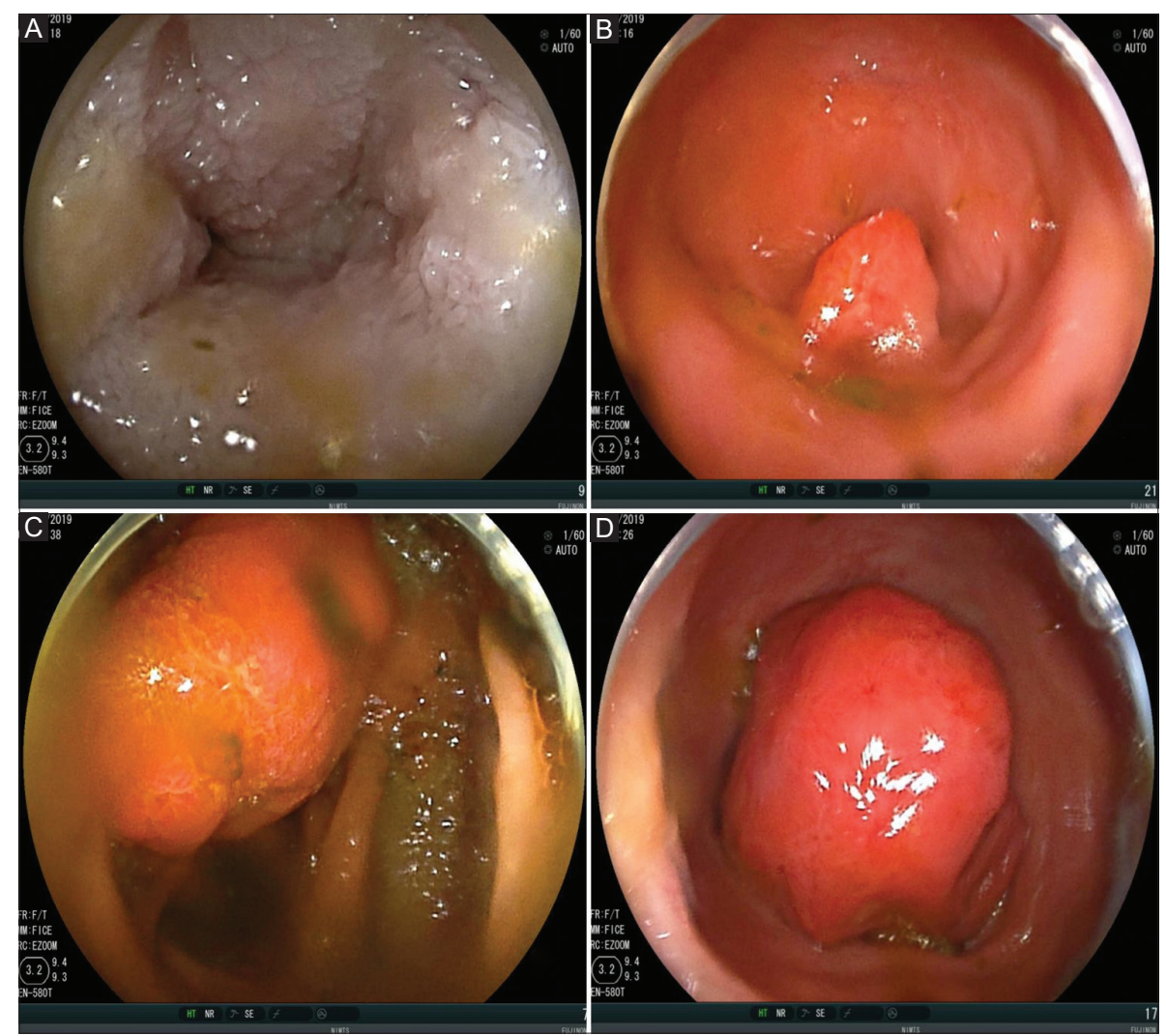

Figure 2 Endoscopic images during retrograde double-balloon enteroscopy. (A) Terminal ileum without endoscopic lesions; (B) small polypoid lesion at $10 \mathrm{~cm}$ from the ileocecal valve (ICV); (C) and (D) a $3.7-\mathrm{cm}$ polyp at a distance of $20 \mathrm{~cm}$ from the ICV, causing intussusception with partial small bowel obstruction 


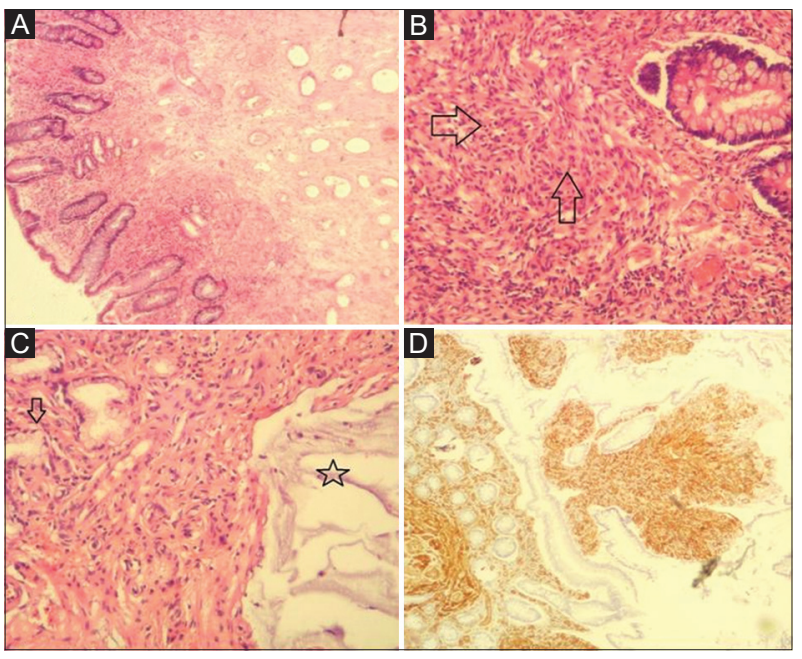

Figure 3 (A) Low-power view of the inflammatory myoglandular polyp. Hematoxylin and eosin ( $\mathrm{H}$ and $\mathrm{E}), \times 40$; (B) stromal hyperplasia with storiform pattern (arrows), $\mathrm{H}$ and $\mathrm{E}, \times 100$; (C) pyloric metaplasia (arrow) and acellular mucin lakes (asterisk). There is no epithelial dysplasia, $\mathrm{H}$ and $\mathrm{E}, \times 100$; (D) $\alpha$ smooth muscle actin immunostain confirms the smooth muscle nature of stromal hyperplasia, 3' $3^{\prime}$ diaminobenzidine chromogen, $\times 40$

inflammatory fibroid polyp, inflammatory myoglandular polyp (IMGP) and prolapse-associated polyps. IMGP, or Nakamura polyp, is an uncommon non-cancerous colorectal, pedunculated polyp, with a smooth red surface, usually located in the left colon [5]. Since the first description in 1992, only 88 cases have been reported [5-10]. Rectal bleeding, positive fecal occult blood test and iron-deficiency anemia are the most common indications for colonoscopy in these patients. There is only one report of IMGP in the terminal ileum causing intussusception, arising from normal mucosa in a non-IBD patient [6]. Histologically, the main differential diagnosis of IMGP includes PeutzJeghers juvenile polyps, where the inflammatory component is significantly less, and inflammatory polyps, where the stromal component is not hyperplastic [5].

Our case is the first description of an inflammatory polyp with both hyperplastic and myoglandular changes in the terminal ileum in an IBD patient. It shows that clinical manifestations with atypical symptoms, especially in cases of well controlled CD, do not always indicate a flare. Clinicians should further proceed to endoscopy and small bowel investigation to exclude other rare causes of abdominal pain. Further research is warranted to identify the pathogenesis of IMPG polyps and to investigate any possible association with chronic inflammation related to IBD or the use of biologic agents among patients with more severe disease.

\section{References}

1. Goldgraber MB. Pseudopolyps in ulcerative colitis. Dis Colon Rectum 1965;8:355-363.

2. Chawla LS, Chinna JS, Dilawari JB, Sood A. Course and prognosis of ulcerative colitis. J Indian Med Assoc 1990;88:159-160.

3. Bockus HL, Roth JL, Buchman E, et al. Life history of nonspecific ulcerative colitis: relation of prognosis to anatomical and clinical varieties. Gastroenterologia 1956;86:549-581.

4. Maggs JR, Browning LC, Warren BF, Travis SP. Obstructing giant post-inflammatory polyposis in ulcerative colitis: Case report and review of the literature. J Crohns Colitis 2008;2:170-180.

5. Nakamura S, Kino I, Akagi T. Inflammatory myoglandular polyps of the colon and rectum. A clinicopathological study of 32 pedunculated polyps, distinct from other types of polyps. Am J Surg Pathol 1992;16:772-779.

6. Griffiths AP, Hopkinson JM, Dixon MF. Inflammatory myoglandular polyp causing ileo-ileal intussusception. Histopathology 1993;23:596-598.

7. Hirasaki S, Kanzaki H, Matsubara M, Suzuki S. Inflammatory myoglandular polyps: a case series of four patients and review of the literature. Gastroenterol Res Pract 2010;2010:984092.

8. Becheanu G, Gheorghe C, Dumbravă M, Serban-Barbu V, Diculescu M. Inflammatory myoglandular colorectal polyps: a series of seven cases and review of literature. Chirurgia (Bucur) 2011;106:613-617.

9. Ahmad A, Lanjewar S, Shafique K, Somma J. Inflammatory myoglandular polyp of the ascending colon: case report and review of literature. Am J Clin Pathol 2016;146:S104.

10. Sánchez-Aldehuelo R, Figueroa-Tubío A, García de la Filia I, et al. Nakamura polyp: an unusual endoscopic finding. Gastroenterol Hepatol 2019;42:34-35. 